\title{
Gene Drive: Can this be the Future of Agricultural Pest Management?
}

\author{
Priyankar Mondal $^{1 *}$, Upasana Mohapatra ${ }^{2}$ and Moumi Ganguly ${ }^{1}$ \\ ${ }^{1}$ Department of Agricultural Entomology, \\ Bidhan Chandra KrishiViswavidyalaya, Mohanpur-741252, India \\ ${ }^{2}$ Department of Plant Biotechnology, University of Agricultural Sciences, \\ Bengaluru-560065, India \\ *Corresponding author
}

\section{A B S T R A C T}

\section{Keywords}

Gene drive, Genetic control, pest management, CRISPR-Cas9, evolution

\section{Article Info}

Accepted:

12 May 2020

Available Online:

10 June 2020
A world free of hunger may be possible when the agricultural production exceeds the global demand for the food. In the era of increasing population, the need for increased food production can be attainable by managing the destructive pests of the agricultural and horticultural crops. The detrimental effects of the pesticides and the attitudes of society towards transgenic crops indicate the researchers to catch out Gene Drive as the substitute method for former methods of crop pest management. In this context, the present narration describes how the self-sustaining CRISPR-based gene drive technology will be the leading technique in the near future for agriculture pest management.

\section{Introduction}

With the enormous increase of human population in last few decades and subsequent cry for food has forced us to practice intensive agriculture with concomitant outbreak of herbivores, weeds and pathogens drastically reducing the yield of cultivated crops. Pest control methods dominated by harmful chemicals left us with toxic residues on food materials and increased resistance of pests against most of the chemicals. This cause the pesticide treadmill to escalate and the ecosystem to suffer from agricultural pollutants (Aktar et al., 2019). But again chemicals cannot be substituted by available physical, agronomical and biological pest control techniques as none of them can rapidly suppress the pests. Here lies the importance of genetic manipulation of pest population.

Gene drive, a CRISPR/Cas9 based gene editing tool is a powerful technology which enable a desirable gene to self-replicate and propagate through a population quicker than any natural mutation. This novel technology has been proposed to combat vector borne 
diseases, control the invasive flora and fauna and even reverse the pesticide resistance (Scudellari, 2019). In the first section of this review, we have summarized the different gene based technologies exploited earlier for controlling pests and in the next section we have discussed the CRISPR-based gene drive in details with its application, constraints and future prospects in relation to agricultural pest management.

\section{Genetic control of agricultural pests}

In agriculture, one of the most revolutionary and widespread gene based technology of recent decades is transgenic crops. These genetically modified crops can express the insecticidal toxins targeting a major pest or a pest complex which would otherwise be extremely difficult to manage by spraying chemicals. For example, within six years of introduction of Bt Cotton in India, there was $24 \%$ increase in cotton yield per acre through reduced pest damage and a 50\% gain in cotton profit among smallholders (Kathage and Quaim, 2012). Transgenic herbicide tolerant crops have completely changed the weed management scenario by re-introduction of broad spectrum non selective herbicides. Glyphosate resistant soybean varieties have been widely adopted by farmers in US which dramatically increased the production and revenue of herbicide industries as well as the crop yield (Owen, 2000). Later the same technology was used to create resistance in maize against glyphosate, glufosinate, 2,4-D and AOPP. The soybean cultivars were also made resistant against glufosinate, dicamba and 2,4-D. With advancement of the gene pyramiding technology such herbicide resistant crops, especially the maize cultivars were stacked with insect resistant Bt traits providing dual resistance (Nandula 2019). Transgenic crops initially performed very well but with time, insects developed resistance against the insecticidal proteins. On the other hand, the herbicide resistant crops are dependent on harmful chemicals with increasing selection pressure on weeds to develop resistance.

Beside such indirect approach to genetically control the pests, several potential methods were also explored for direct genetic manipulation of pest population. The first such technique to deploy for area wide pest management was the 'sterile insect technique'(Knipling, 1985). This technology (SIT) relies on irradiation (X-ray, $\gamma$-ray) of mass reared insects (preferably males) to make them sterile while retaining the mating ability. These sterile males are systematically released in the wild where they compete with wild males to fertilize the wild females and produce inviable embryos (due to dominant lethal mutations in irradiated male gametes) resulting into sharp decline of the population within a few generations.

The SIT has been successful in controlling a number of high-profile insect pests, including fruit flies (Mediterranean fruit fly, Mexican fruit fly, oriental fruit fly, melon fly); tsetse fly; screwworm; moths (codling moth, pink bollworm, false codling moth, cactus moth, and the Australian painted apple moth); and mosquitoes (Hendrichs and Robinson, 2009). The burden to handling a large population in laboratory, isolating them based on sex, irradiation related fitness costs (reduced competitiveness, internal tissue damage) and essentiality of single mating habit in females of the target insect are some of the major drawbacks to apply this technique.

A suitable alternative of non-transgenic SIT is a recently introduced transgenic technology named as "release of insects carrying a dominant lethal" (RIDL) gene technique (Thomas et al., 2000). This approach relies on a specific strain of mass reared target insect 
which carries a dominant, sex specific lethal gene activated by expression of a tetracycline repressible transactivator fusion protein (tTA) which is again controlled by a female specific, embryo specific promoter (yp3). Tetracycline, generally added to the diet of insects in the rearing facilities, prevents the expression of tTA allowing the insects to live and grow there.

Once the transgenic insects are released in the environment the expression of the lethal gene (in absence of tetracycline) causes mortality in the early developmental stages of the heterozygous progeny but does not affect the viability of the transgenic parents resulting an effective control of the wild pest population (Black et al., 2011).

'Incompatible insect technique' (IIT) is another genetic control technique which relies neither on irradiation, nor transgenesis. This approach depends on Woolbachia infected males with cytoplasmic incompatibility mating with uninfected females in wild and producing inviable off springs (Zabalou et al., 2009). The mechanism of CI has not yet been well understood at the molecular level.

However, few authors suggest that Wolbachia somehow modifies the male chromosomes during spermatogenesis which eventually alters chromosome kinetics during the first mitotic divisions. This may result into mitotic asynchrony and death of the embryo (Tram and Sullivan, 2002).Heat shock can affect the Woolbachia density, transmission and subsequently Woolbachia-related effects. It has been shown that in Culex and Drosophila the expression of CI is negatively correlated with age of males (Reynolds and Hoffman, 2002).

SIT, RIDL and IIT are essentially described as 'self-limiting mechanisms'relying on inundativerelease of modified insects that mate with local population resulting into suppression of the population through lethal matings. As these methods depend on lethality, they do not persist and spread through populations and proportion of mating between introduced and the wild population becomes a major limiting factor for their application (Neve, 2018).

\section{Gene drive, a hijacking tool}

A gene drive may be defined as a package of selfish genetic elements capable of propagating and sustaining itself throughout a population without affecting the fitness of individuals carrying it. In simple language, it is a modified gene designed to spread through a population at a rate (nearly 100\%) higher than the normal rate $(50 \%)$ of inheritance. This CRISPR based technology can completely redefine the genome of an entire species. In this section we will discuss how this technology works in an organism.

The gene drive contains three important elements: i) a gene encoding bacterial Cas9 nuclease enzyme, ii) a gene encoding the guide RNA which targets a particular site in the genome and iii) the altered or modified gene which will modify the phenotype of the target insect (Figure 1)(Courtier- Orgogozo et $a l ., 2017)$. Once a gene drive is engineered into an animal's genome, the animal's offspringwill inherit the drive on one chromosome and a normal gene from its other non-engineered wildparent. During early development, the Cas9 will automatically cut the same site on the homologous chromosome and insert the edited gene through homologous recombination pathway. Thus the offsprings become homozygous for the respective gene drive (CRISPR-Cas9+edited gene complex) and can transmit the same through subsequent generations like a mutagenic chain reaction (Gantz\& Bier, 2015). 
As a result, within a few generations, the complete population of a species is edited with the desired gene (Figure 2). In contrast to the 'self-limiting mechanisms' discussed earlier CRISPR-based gene drive technology is a 'self-sustaining mechanism' with an ability to sustain and enhance the transmission through subsequent generations of the target species. Even, invasion of wild individuals can no longer dilute the gene and disrupt the system. Through this technology we can theoretically hijack the evolutionary trajectory of a species (Scudellari, 2019).

\section{Application of gene drive for agricultural pest management}

Any desirable DNA sequence (gene) can be inserted into the gene drive, which enables a wide range of application of this technology. With respect to agriculture, we can use this technology to control insect vectors of plant and animal diseases, suppress the population of invasive species, restore the susceptibility in resistant pest populations, enhance biological control by manipulating infectivity or pathogenicity of microbes or host searching ability of predators and parasitoids, increase the foraging efficiency of pollinators, reduce the population of different invertebrate and vertebrate pests inserting genes which directly or indirectly facilitates overall reduction in herbivory.

So far the technology has been successfully tested in lab reared mosquitoes, yeasts, flies, mice and even human cells. Theoretically, population of any sexually reproducing species can be manipulated by gene drive. Compared to available efficient pest management strategies, this new technology is quite cheaper in spite of being extremely precise on target insect(Courtier- Orgogozo et al., 2017). According to estimates, it may take US\$1,000 to construct a gene drive organism whereas, to develop and release a new pesticide US\$ 40,000-2,250,000 may be consumed (Barnard, 1958).

\section{Constrains of gene drive- the molecular and ecological perspectives}

There is no doubt that gene drive as a technology can dramatically change the agricultural scenario with sharp increase in production and concomitant upsurge in economy but every good thing comes with a cost and in case of gene drive these costs are speculative yet can enormously affect the ecosystem. In this section we shall discuss the molecular and ecological constraints of this technology.

Larger genome with similar off-target sequences can potentially reduce the efficiency of gene drive but it should not be a constraint as most insects have smaller genomes compared to the vertebrate species which have undergone successful trials of this technology. Self-sustainability of gene drive depends on successful copying and pasting of the inserted gene in the same locus of homologous chromosome through homologous recombination pathway. In this pathway only the desired gene present in one chromosome is used as a template for repairing the cut DNA in the homologous chromosome.

The cut DNA can also be repaired by nonhomologous end joining pathway in which broken ends are directly ligated without copying the desired gene and as a result, homozygosity for the gene drive is not achieved. The relative frequency of these two pathways for repairing DNA may vary within species which can limit the success of this technology for different pest species (Neve, 2018). Experiments resulted into more than $90 \%$ success in mosquitoes but less than $78 \%$ success in fruitflies (Windbichler et al., 2011; Chan et al., 2011). 


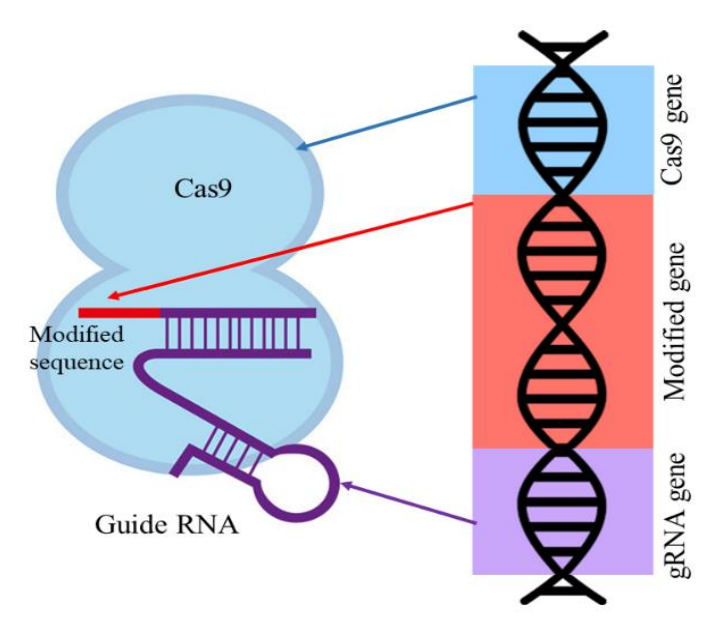

Figure.1 Components of a CRISPR/Cas9 based gene drive

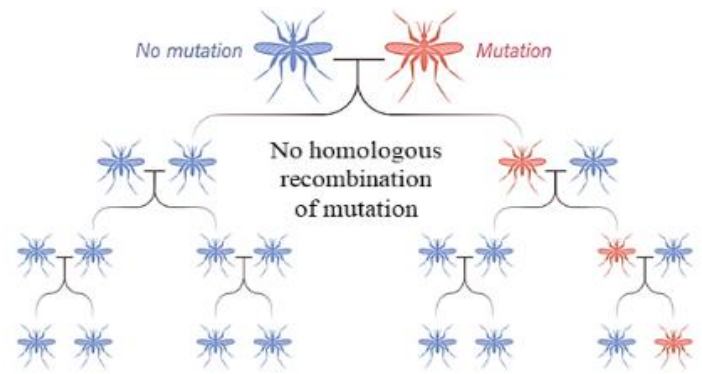

a) Normal inheritance

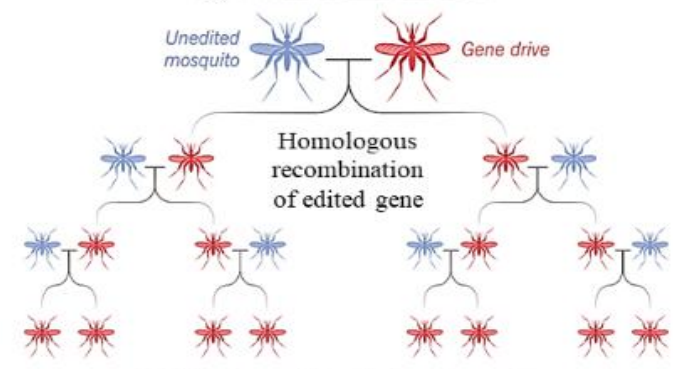

b) Gene drive inheritance

Figure.2 Difference between the slow normal inheritance and faster gene drive inheritance (Adapted from Scudellari, 2019)

Hijacking the natural course of evolution is not so easy and that is why any gene based technology suffers from counter-adaptation by the target organisms. Gene drive frequency may gradually be decreased over multiple generations owing to mutations at target site of the genome which remains unrecognized by the Cas9 (Hammond et al., 2017). This can be overcome by selecting more conserved genes which do not accumulate rapid mutations as alternation of sequence in such genes can cost drastic reduction in fitness (Scudellari, 2019).

Even if we overcome the molecular limitations, then comes the ecological impact which is completely speculative and obscure as none of the trials were performed in wild. 
The rate at which gene drives can spread depends on several life history parameters and demographic traits of the species. Some suitable situations for quicker and efficient propagation of gene drives were stated by Neve (2018): i) the introduced population of engineered species should preferably be larger than the established population, ii) there should not be any fitness cost associated with the drive iii) the generation time of target species should be shorter, iv) target species must reproduce through sexual means (outcrossing), v) in case of plants, pollen or seeds containing the gene drive can enhance its spread through dispersal.

Implementation of gene drive for managing agricultural pests may cause immediate suppression but can also cause long term ecological effects which may take few decades to appear. 'Pest' is a relative term indicating a population rather than a species which causes economic loss but that particular species is an integral part of an ecosystem. It may be food for many beneficial organisms or serve as pollinators of both cultivated and non-cultivated plants. As per current experimental results, if not managed properly a gene drive can cause total eradication of a species which may drastically affect the ecosystem by disrupting food chain and ecosystem services.

Thus, it is concluded that the CRISPR based gene drive is a technology confined in the laboratories and waiting to be released in the environment. As we have already discussed, once released for commercial purpose, this particular technology can revolutionize the current medical and agricultural scenarios. Of course, there are a number ecological constraints with potential ethical restrictions associated with adoption of this technique but those can be managed by suitable regulations of such gene based technologies. The agrifood industries will particularly come forward to adopt this cheap yet effective technology but generalizing the use of CRISPR based gene editing toolscan cause huge ecological catastrophes as varieties of mutation released by different organization in an ecosystem can jeopardize it completely. To date, handfuls of biologists have mastered this sophisticated technology and this technical feasibility in future will be one of the prime requirement for effective utilization of gene drive. Laboratory trials with major agricultural pests such as stem borers, fruit flies, armyworms, weevils, aphids, mites, major plant pathogens and major weeds of different agroecosystem should immediately be performed to observe the practical impact on these organisms. Ecologists should also come together with molecular biologists to model the impact of the technology on different ecosystems before practically releasing for commercial purpose.

\section{References}

Aktar, W., Sengupta, D., \& Chowdhury, A. (2009). Impact of pesticides use in agriculture: their benefits and hazards. Interdisciplinary toxicology, 2(1), 1-12.

Barnard OC. (1958) Pesticide Development Costs.Journal of Agricultural and Food Chemistry, 6 (7), 512-513

Black IV, W. C., Alphey, L., \& James, A. A. (2011). Why RIDL is not SIT. Trends in parasitology, 27(8), 362-370.

Chan, Y. S., Naujoks, D. A., Huen, D. S., \& Russell, S. (2011). Insect population control by homing endonuclease-based gene drive: an evaluation in Drosophila melanogaster. Genetics, 188(1), 33-44.

Courtier-Orgogozo, V., Morizot, B., \&Boëte, C. (2017). Agricultural pest control with CRISPR-based gene drive: time for public debate. EMBO reports, 18(6), 878-880.

Gantz VM and Bier E, The mutagenic chain reaction: a method for converting 
heterozygous to homozygous mutations. Science 348:442-444 (2015).

Gantz, V. M., \& Bier, E. (2015). The mutagenic chain reaction: a method for converting heterozygous to homozygous mutations. Science, 348(6233), 442-444.

Hammond, A. M., Kyrou, K., Bruttini, M., North, A., Galizi, R., Karlsson, X., \& Nolan, T. (2017). The creation and selection of mutations resistant to a gene drive over multiple generations in the malaria mosquito. PLoS genetics, 13(10), e1007039.

Hendrichs, J., \& Robinson, A. (2009). Sterile insect technique. In Encyclopedia of Insects (pp. 953-957). Academic Press.

Kathage, J., \&Qaim, M. (2012). Economic impacts and impact dynamics of $\mathrm{Bt}$ (Bacillus thuringiensis) cotton in India. Proceedings of the National Academy of Sciences, 109(29), 1165211656.

Knipling, E. F. (1985). Sterile insect technique as a screwworm control measure: the concept and its development. In Symposium on eradication of the screwworm from the United States and Mexico. Misc. Pub. Entomol. Soc. Am (Vol. 62, pp. 4-7).

Nandula, V. K. (2019). Herbicide Resistance Traits in Maize and Soybean: Current Status and Future Outlook. Plants, 8(9), 337.

Neve, P. (2018). Gene drive systems: do they have a place in agricultural weed management? Pest management science, 74(12), 2671-2679.
Owen, M. D. (2000). Current use of transgenic herbicide-resistant soybean and corn in the USA. Crop Protection, 19(8-10), 765-771.

Reynolds, K. T., \& Hoffmann, A. A. (2002). Male age, host effects and the weak expression or non-expression of cytoplasmic incompatibility in Drosophila strains infected by maternally transmitted Wolbachia. Genetics Research, 80(2), 79-87.

Scudellari, M. (2019). Hijacking evolution. Nature, 571(7764), 160-162.

Thomas, D. D., Donnelly, C. A., Wood, R. J., \&Alphey, L. S. (2000). Insect population control using a dominant, repressible, lethal genetic system. Science, 287(5462), 2474-2476.

Tram, U., \& Sullivan, W. (2002). Role of delayed nuclear envelope breakdown and mitosis in Wolbachia-induced cytoplasmic incompatibility. Science, 296(5570), 1124-1126.

Windbichler, N., Menichelli, M., Papathanos, P. A., Thyme, S. B., Li, H., Ulge, U. Y., \&Crisanti, A. (2011). A synthetic homing endonuclease-based gene drive system in the human malaria mosquito. Nature, 473(7346), 212-215.

Zabalou, S., Apostolaki, A., Livadaras, I., Franz, G., Robinson, A. S., Savakis, C., \&Bourtzis, K. (2009). Incompatible insect technique: incompatible males from a Ceratitis capitata genetic sexing strain. Entomologia Experimentalis et Applicata, 132(3), 232-240.

\section{How to cite this article:}

Priyankar Mondal, Upasana Mohapatra and Moumi Ganguly. 2020. Gene Drive: Can this be the Future of Agricultural Pest Management?. Int.J.Curr.Microbiol.App.Sci. 9(06): 131-137. doi: https://doi.org/10.20546/ijcmas.2020.906.017 